\title{
DESIGN AND DEVELOPMENT OF AN IOT BASED MULTI TERRAIN
}

\section{HUMANOID ROBOT FOOT}

\section{A. JOSIN HIPPOLITUS \& AYUSH OBEROI}

SRM Institute of Science and Technology, Chennai, Tamil Nadu, India

\begin{abstract}
A major leap in the purview of robotics will be seen when a humanoid robot is invented. The foot designed for the humanoid was not terrain friendly. What makes the human foot more flexible and sturdy is the number of bones present in it. This was a challenge in humanoid robot foot design. The humanoid robot foot here is made to be terrain friendly and sturdy at the same time. When in motion, the foot physically represents the human foot with a curve in its bottom, which helps in its balancing as well as maintaining the center of gravity. Toes which can be folded are made to increase the stability of the robot in motion. The foot is designed using fusion 360 and has been $3 D$ printed. It can be operated via internet from a mobile device. A smart phone with client and server operating system through a Node MCU module was used to operate it wirelessly.
\end{abstract}

KEYWORDS: Robotic Foot, IOT in Robotics, Humanoid Foot \& Multi-Terrain Robotic Foot

Received: Aug 01, 2018; Accepted: Aug 21, 2018; Published: Oct 23, 2018; Paper Id.: IJMPERDDEC201816

\section{INTRODUCTION}

There have been many researchers, who have studied and worked upon flat robotic foot, which lack in stability on uneven rocky terrain. The design of flexible robot foot in field environment is highly significant to its performance in terms of working efficiency and anti-sinking. The mechanical design was established in accordance with the structure and character of human's foot and would replicate the motions similar to that of a human foot. Working mechanism is based upon human nervous system. At present, the basic idea is to make a sample similar to just like actual working Human foot, which has perfect hold and grip on any kind of surface.

Given the overnight developments and improvements in robotic technology around the world, significant robotic applications were developed to better the quality of our lives. The International Organization for Standardization describe a robot in ISO 8373 as "an automatically controlled, reprogrammable, multipurpose, manipulator programmable in three or more axes, which may be either fixed in place or mobile for use in industrial automation applications." Actual robots are constructed that seem impossible for the public as the equipment and the skill need is exorbitant and difficult to learn. Constructing prototype of a real robot certainly is still possible to display the effectiveness of the desired application.

\section{Challenges}

Since research on measurable depiction of flat foot describes that uttermost biped robots walk with flat feet that arrive parallel to the ground, robots were able to perform certain challenges as implemented. When walking, the robot's centre of mass had a lateral movement of $50 \mathrm{~mm}$ (compared to just $30 \mathrm{~mm}$ for a human). And, it couldn't copy a human's foot rotation (approximately 12 degrees) due to a lacking of yaw joint in its ankle; a 
problem derived by the old joint setup. While designing the prototype, the yaw rotation will be provided by a motor situated at the top of the shin, just below the knee. The two motors normally situated in the ankle for pitch, and rolls aren't used in favour of a linear parallel link mechanism, which cut short precious millimetres off the lower leg's perimeter.

\section{SCOPE OF THE RESEARCH}

It can be clearly claimed that a range of necessary capabilities are in need to be present for flexible robot foot to be useful. For a robot to operate safely, i. e. it should stay far from danger such as obstacles or operating conditions hazardous to the robot itself (e. g. Downhill or stairs), and it should pose no damage to humans in the proximity of the robot.

There are many applications of flexible robot foot, and their importance in medical prosthetics as well as foot locomotion on multi terrain continues to grow. Flexible foot can be used for performing locomotion as humans in case of robots. Increasingly, they play an economic role in replacing human damaged leg. A major aspect of multi terrain locomotion of flexible foot is projected. Such innovative locomotion is hard to achieve with traditional flat foot, since those techniques desire to balance at uneven surfaces with changing COG at each point. Yet by definition, abnormalities are rare, so still flat feet are commonly used to balance robot. The main focus of flexible foot is to balance and walk on multi terrain land.

The robotic foot normally used has a flat bottom surface, which will not suit all conditions. While developing a robot which can move in any terrain, this kind of design becomes incompatible. Thus, designing the flexible foot with fingers which suits all terrain and adapt any kind of environment is essential. The fingers present in the foot provide grip for a stance and gait of a robot.

\section{LITERATURE SURVEY}

Heel-Strike and Toe-Off Motions Optimization for Humanoid Robots Equipped with Active Toe Joints (2018)

Majid Sadedel et al have been working with humanoid robot's foot. By experimenting with the heel-strike and toe-off motions, they identified that heavier is the toe link, lower is the instability and slippage occurrence possibility.

\section{Development of a Biped Robot with Toes to Improve Gait Pattern (2003)}

Cheol Ki Ahn et al have done an analysis on a biped robot. They concluded that, the toe joint affects gait pattern generation and helps in natural walking, similar to the human gait or faster walking like running.

\section{Robotic Walking In Natural Terrain: Gait Planning and Behavior-Based Control for Statically-Stable Walking Robots (1996)}

David Wettergreen stated that small obstacles can be avoided by making discrete contacts and passing up undesirable footholds.

\section{Stepping for Balance Maintenance Including Push-Recovery (2017)}

Jerry Edwin Pratt et al states that, the direction of forces on the center of mass could be shifted by modifying its base of support. For a humanoid to balance a push or pull, it will take a very fast step or two to an appropriate place to recover balance. They also discussed the center of pressure and angular momentum-based balance strategies together with stepping. 


\section{DESIGN EVOLUTION}

Design inspiration came from Human's foot, which is capable of performing various functions with great stability. Flexible robot foot is designed by studying the structural characteristics of human foot, and its ability to walk on multi terrain surface. Flexible foot is divided into 3 parts and each part was designed in fusion 360 . After few designs, we achieved a final design, which appeared just like human foot. Design was fabricated using 3D printing technique and then assembled together. The design was to avoid small obstacles by placing the foot in the appropriate places and by making discrete contacts [3]. Hence, the bottom of the foot has an arch, so that small obstacles like a stone can be avoided by not compromising on the stability, and Centre of Pressure (COP).

\section{Early Stage Design}

In the early stage design, the humanoid foot has toes which are fixed. As fixed toes have no effect, the toes had to be flexible which increases stability. The toe joint affects the gait pattern much. [2]
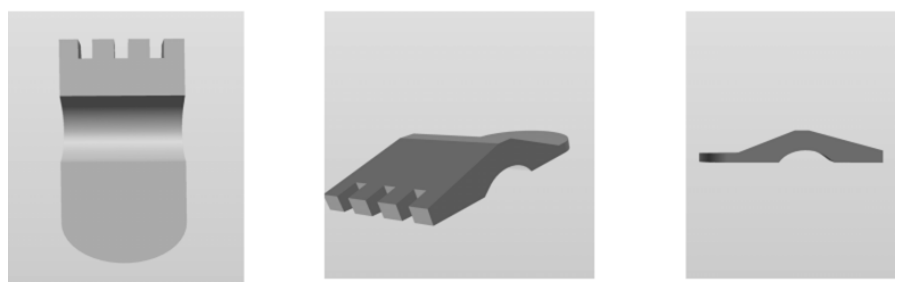

Figure 1: Early Stage Design

\section{Intermediate Design}

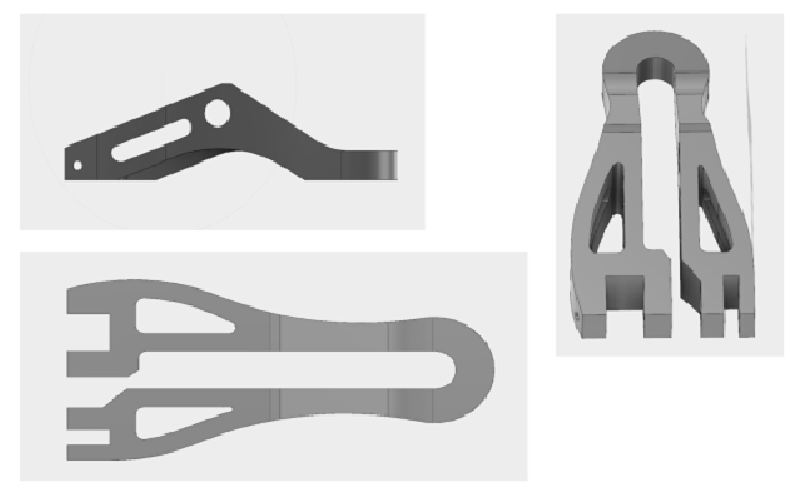

Figure 2: Intermediate Design

In the intermediate design, a hinge like structure is designed, so that the foot can have a pitch motion along that axis. The hinge for the leg to connect with the foot is placed such that, the center of pressure (COP) will never fall outside the foot, making it more stable. [4], which is shown in Figure 7

\section{Design of Toes and Big Thumb}

The Toes and Big Thumb are designed so that slippage could be avoided [1]. And hence, the toes and big thumb are made thicker and firmer 


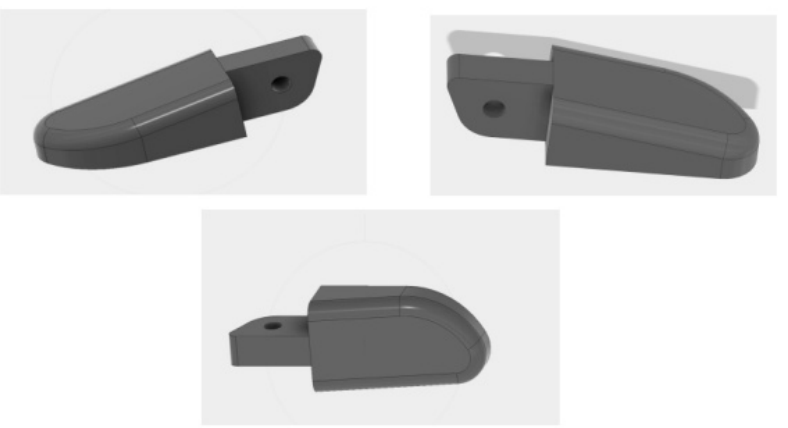

Figure 3: Thumb Design

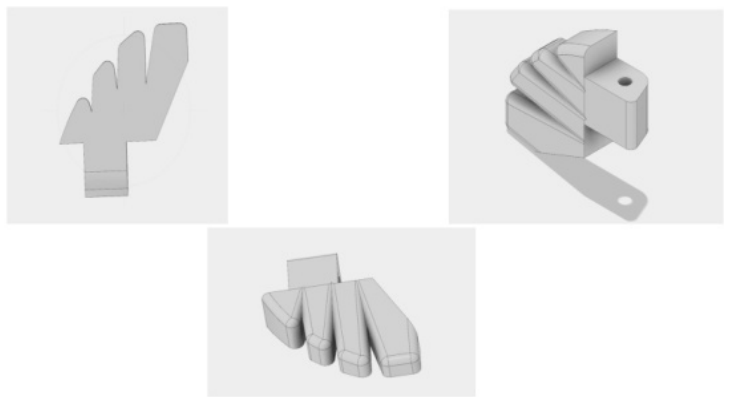

Figure 4: Fingers Design

\section{Design of Shaft}

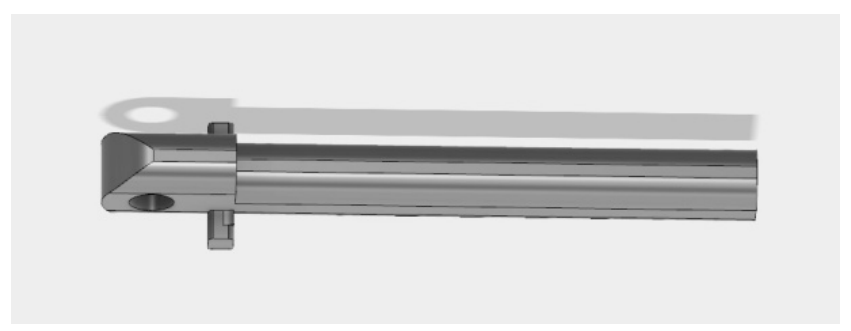

Figure 5: Top View of Shaft

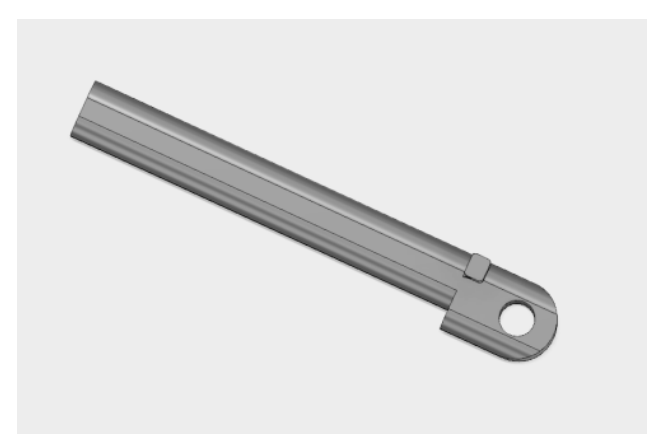

Figure 6: Right Side View of Shaft 


\section{Ultimate Design}

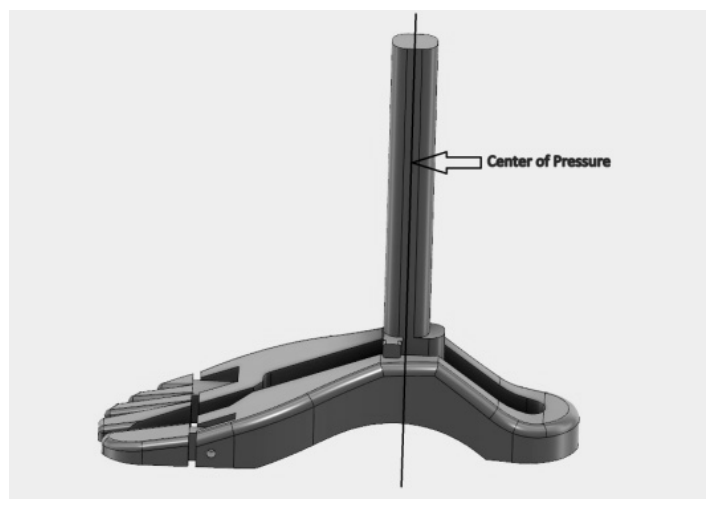

Figure 7: Side View of Ultimate Design

Servo motors are used for actuation of fingers and foot, and are connected to microcontroller. Node MCU acts as the server, which responds to the signals provided by the remote client, which is the smart phone. It is powered by a $12 \mathrm{v}$ battery or an USB power source. The servos used are of two different types i. e. Sg-90 \& Sg-3003. Node MCU uses firmware, which works on the ESP8266 WiFi SoC from Espressif Systems, and hardware which is based on the ESP-12 module. The power bank is rated for $10.4 \mathrm{Ah} 5 \mathrm{~V}$.

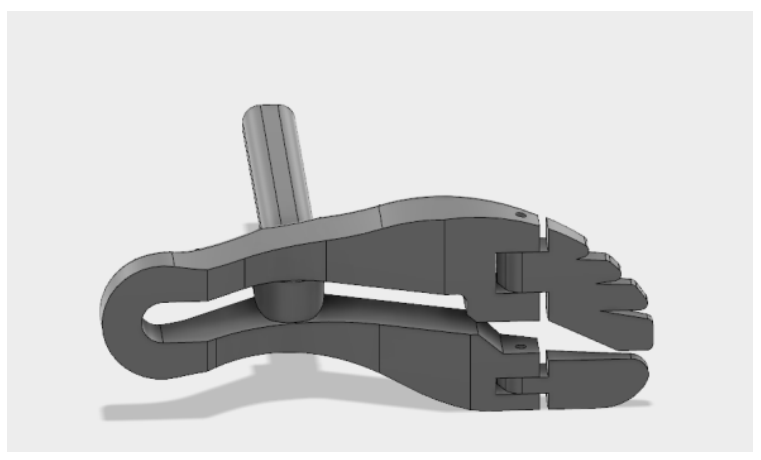

Figure 8: Bottom View of Ultimate Assembly

\section{WORKING AND OPERATION}

The foot consists of 3 servo motors, connected to 3 output pins of Node MCU. Two less torque servo motors are attached at fingers and foot joint for creating a pitch movement in the Robot Foot. And, one high torque servo motor is attached at the main shaft.

$\mathrm{S} 1$ servo motor $1 \rightarrow \mathrm{D} 3$ port, $3 \mathrm{v}$ power supply, GND
$\mathrm{S} 2$ servo motor $2 \rightarrow \mathrm{D} 2$ port, $3 \mathrm{v}$ power supply, GND
$\mathrm{S} 3$ servo motor $3 \rightarrow \mathrm{D} 4$ port, $3 \mathrm{v}$ power supply, GND

Node MCU creates a Local server Wi-Fi, which can be connected to any of the local client, based on similar IP address. Interface is developed using html, and a web page at local server is used to operate the various motors. Motors are connected to various GPIO terminals on Node MCU and are powered by a $5 \mathrm{~V}$ power source; each motor requires 3 Volts to operate. Commands are given using a local client web interface and are received by the server. Then, the server responds to the commands respective to the pin number, in which the motor is connected. 


\section{Control Panel}

It has 4 different types of operational function and each function is performed according to the desired positional requirement. It can also work in combination like fingers up, plus foot down or both up plus big thumb up etc.

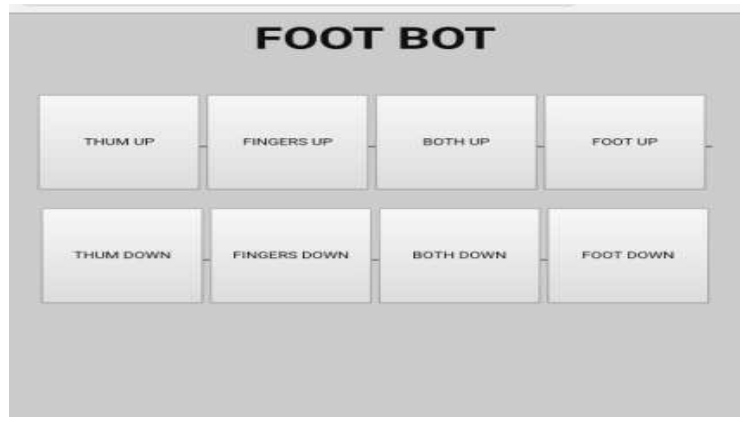

Figure 9: Control Panel

\section{Modes of Operation}

\section{Thumb and Fingers Operation}

Thumb is connected with servo motor S2, which operates up and down motion based upon retraction. Acrylic threads are tied, which act nerves to pass signals. 4 Fingers are set as a group and tied using acrylic tried together, operate using servo motor $\mathrm{S} 1$ following the similar mechanism as thumb operation.
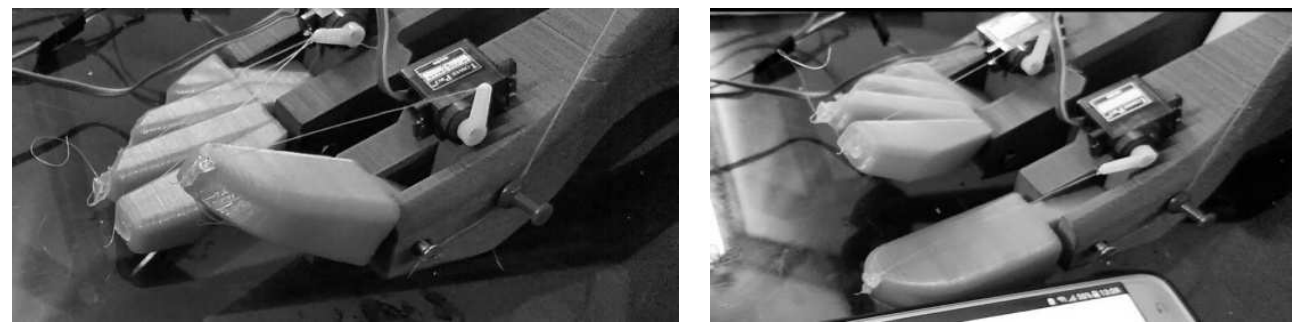

Figure 10: Thumb and Fingers Operation

\section{Both up}

Both fingers and thumb are stretched i. e. both the motors are active.

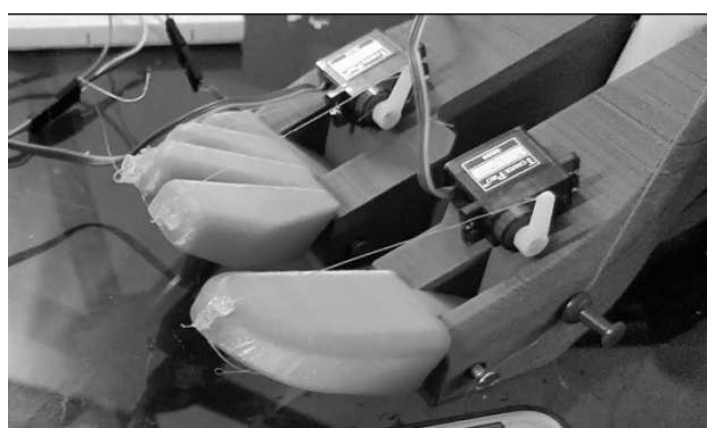

Figure 11: Both Operations

\section{Foot up and down}

Main shaft is attached with a servo motor S3, which operates on the rack and pinion mechanism and the motor works on high and low state. 


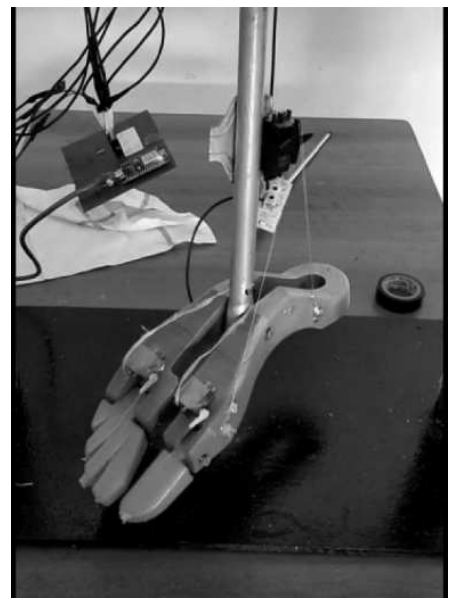

Figure 12: Foot Up and Down

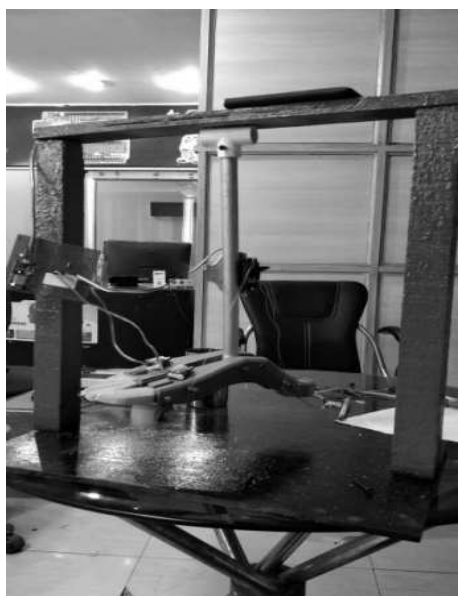

Figure 13: Final Setup

\section{CONCLUSIONS}

The main purpose of this project is to develop a flexible robot foot, which is capable of withstanding multi terrain surfaces and control using wireless technology IOT. The locomotion was implemented using Node MCU controller, which consists of Wi-Fi module for client server communication. 3 servo motors connect with Node MCU for movement of various parts. User can control the movement of robot using access to the Wi-Fi server and desired IP address. Servo motors are programmed according to the position required. The project gave a closer approach to the structural design of human foot. The prototype of this project has been successfully completed, where the flexible foot can move according to the user instructions from the client web interface. The idea of implementing IOT is too look forward in new level wireless control over the communication between client and server. The conclusions and result achieved from the project is a light and effective weight flexible foot robot, which delivers a flexible and precise antagonistic locomotion on a smooth or uneven terrain. This can be useful for precision activity, as it achieves the desired position according to the set position and holds on until reverse signals provided by the user. Robot Foot consists of flexible fingers, thumb and a main shaft is attached to the frame, which displays the prototype of the actual robot.

\section{REFERENCES}

1. Sadedel, M., Yousefi-Koma, A., Khadiv, M., \& Iranmanesh, F. (2018). Heel-strike and toe-off motions optimization for humanoid robots equipped with active toe joints. Robotica, 36(6), 925-944. doi:10.1017/S0263574718000140

2. Cheol Ki Ahn, Min Cheol Lee and Seok Jo Go, (2003)"Development of a biped robot with toes to improve gait pattern," Proceedings 2003 IEEE/ASME International Conference on Advanced Intelligent Mechatronics (AIM 2003), 2003, pp. 729734 vol. 2 .

3. Bharath, L., \& Himanth, M. A Comparative Study Between The Successive Screw Displacement And Quaternion Based Methods Used In Forward Kinematics Of Serial Robot Manipulator.

4. Wettergreen, David. (1997) "Robotic walking in natural terrain Gait planning and behavior-based control for statically-stable walking robots." Carnegie Mellon University Pittsburgh, PA, USA.

5. Pratt, Jerry \& Bertrand, Sylvain \& Koolen, Twan. (2017). Stepping for Balance Maintenance Including Push-Recovery, Introduction to Humanoid Balance. 1-48. 10.1007/978-94-007-7194-9_41-1. 
